\title{
IDENTIFICATION OF CENTRAL CHOLINERGIC NEURONS CONTAINING BOTH CHOLINE ACETYLTRANSFERASE AND ACETYLCHOLINESTERASE AND OF CENTRAL NEURONS CONTAINING ONLY ACETYLCHOLINESTERASE ${ }^{1}$
}

\author{
FELIX ECKENSTEIN² AND MICHAEL V. SOFRONIEW
}

Department of Neurochemistry, Max Planck Institute for Psychiatry, D-8033 Martinsried-Munich, Federal Republic of Germany and Department of Human Anatomy, University of Oxford, Oxford OX1 3QX, England

Received February 28, 1983; Revised May 23, 1983; Accepted May 25, 1983

\begin{abstract}
Neurons in the rat central nervous system (CNS) were examined for their content of both the acetylcholine-synthesizing enzyme, choline acetyltransferase (ChAT; acetyl-CoA, choline $O$-acetyltransferasc, EC 2.3.16), and the transmitter-degrading enzyme, acetylcholinesterase (AChE). ChAT was localized immunohistochemically and AChE was localized histochemically in normal, colchicinetreated, or diisopropylfluorophosphate (DFP)-treated rats, either in neighboring sections by standard procedures or simultaneously in the same sections by immunofluorescence for ChAT, followed by photography, followed by histochemistry for $\mathrm{AChE}$, followed by brightfield rephotography of the same neurons. This combination of fluorescence and brightfield procedures allows both to be carried out at maximum intensity without fear of interference and enables the unambiguous visualization of all neurons that might be expected with either procedure. Based on previous examination of singly stained neighboring sections, five CNS areas were examined in detail in DFP-treated rats using the simultaneous procedure for localizing both ChAT and AChE in the same neurons in the same sections. Several hundred neurons were thus examined in each area. These areas were the caudate putamen, nucleus basalis of Meynert, medial septum, nucleus of the diagonal band of Broca (vertical and horizontal limbs), and zona incerta. In the caudate putamen and nucleus basalis magnocellularis of Meynert, all ChAT-positive neurons contained AChE and vice versa. In the medial septum and nucleus of the diagonal band of Broca, all ChAT-positive neurons contained $\mathrm{AChE}$, but these neurons containing both $\mathrm{ChAT}$ and $\mathrm{AChE}$ were intermingled with neurons positive only for AChE. Throughout these two areas, approximately $12 \%$ of the intensely AChE-positive neurons did not contain ChAT. In the zona incerta, no neurons positive for ChAT but many positive for AChE were regularly observed. Other areas containing intensely AChE-positive but not ChATpositive neurons, as revealed in neighboring sections stained for AChE or ChAT, included the hypothalamic arcuate and dorsomedial nuclei, the lateral posterior hypothalamus, and the substantia nigra. All ChAT-positive neurons in the areas examined always contained AChE, but this AChE staining could not always be characterized as intense.

These findings indicate that in those areas examined, central cholinergic neurons contain both ChAT and AChE. In addition, presumed noncholinergic neurons exist which contain AChE but not $\mathrm{ChAT}$, and in some areas these two types of aChE-positive neurons may be intermingled.
\end{abstract}

Although acetylcholine was the first molecule identified to act as a neurotransmitter, the study of the mor-

\footnotetext{
${ }^{1}$ This work was supported in part by the National Institute of Neurological and Communicative Disorders and Stroke, National Research Service Award 1 F32 NSO6959. The editorial assistance of P. Campbell is gratefully acknowledged. We thank Z. Henderson for helpful advice regarding the histochemical procedure for acetylcholinesterase.

${ }^{2}$ Present address: Department of Neurobiology, Harvard Medical School, 25 Shattuck Street, Boston, MA 02115.
}

phology of central cholinergic neurons is far less advanced than that of many other neurons producing other transmitter candidates (Fibiger, 1982). This is in part due to the lack of an in situ method for directly localizing acetylcholine, necessitating the use of other markers for the anatomical identification of cholinergic neurons (Fibiger, 1982). The first attempt in this direction was the histochemical localization of acetylcholinesterase (AChE), the transmitter-degrading enzyme, originally described by Koelle and Friedenwald (1949), which has 
now been developed into a routine and widely used procedure (Butcher, 1978; Fibiger, 1982). However, it has become suspect that $\mathrm{AChE}$ is not a specific marker labeling only cholinergic neurons, since the enzyme is present in different populations of monoaminergic neurons and in non-neuronal structures (Rossier, 1977; Butcher, 1978; Fibiger, 1982). At present, the acetylcholine-synthesizing enzyme, choline acetyltransferase (ChAT; acetyl-CoA, choline $O$-acetyltransferase, EC 2.3.16), represents the most useful and valid marker for cholinergic neurons (Fonnum, 1975). Since the available histochemical method for visualizing this enzyme is, unfortunately, not reliable (see Butcher, 1978; Fibiger, 1982), efforts have been concentrated in a number of laboratories on immunohistochemical detection methods for ChAT. Studies of this nature have given rise to a good deal of controversy centering around the purity of the ChAT preparations used to generate antisera and the specificity of the immunohistochemical staining obtained with these antisera (see Rossier, 1981). We have recently developed specific preparations of ChAT for immunohistochemical identification of cholinergic neurons (Eckenstein et al., 1981; Eckenstein and Thoenen, 1982) and have described the topography of cholinergic neurons in the rat forebrain (Sofroniew et al., 1982). Comparison of our findings with the known distributions of AChEcontaining neurons (Jacobowitz and Palkovits, 1974) indicated that several areas rich in AChE-positive neurons did not contain ChAT-positive neurons. In view of the widespread use of histochemical staining procedures for AChE to study "cholinergic" neurons and the abovementioned doubts as to the specificity of AChE as a marker for cholinergic neurons, this study was undertaken to analyze several populations of AChE-positive neurons for ChAT immunoreactivity. This was done by indirect immunofluorescent staining for ChAT followed by the thiocholine histochemical procedure for AChE in the same tissue sections. Several representative areas of the rat brain were examined in detail based on observations of ChAT or AChE staining in neighboring sections. The procedure used has allowed the discrimination between neurons containing both ChAT and AChE and those containing only AChE. In addition, it has enabled us to test the hypothesis that all cholinergic, i.e., ChATcontaining, neurons express AChE.

\section{Materials and Methods}

For localization of ChAT and AChE in neighboring sections, four normal Wistar rats and two Wistar rats injected with $100 \mu \mathrm{g}$ of colchicine in $20 \mu \mathrm{l}$ of $\mathrm{H}_{2} \mathrm{O}$ into the lateral cerebral ventricle and allowed to survive for $36 \mathrm{hr}$ were perfusion fixed under anesthesia using buffered $4 \%$ paraformaldehyde. The brains were removed and $50-\mu \mathrm{m}$ Vibratome sections were collected. Alternate sections were stained for ChAT, using the immunoperoxidase procedure as previously described (Sofroniew et al., 1982), and for AChE, using the thiocholine procedure as described by Lewis and Shute (1969).

For simultaneous localization of ChAT and AChE in the same neurons in the same tissue sections, six Wistar rats were injected intramuscularly with $2 \mathrm{mg} / \mathrm{kg}$ of diisopropylfluorophosphate (DFP) and $2 \mathrm{mg} / \mathrm{kg}$ of atropine according to the method of Woolf and Butcher (1981), allowed to survive for $8 \mathrm{hr}$, and perfusion fixed under anesthesia using buffered $4 \%$ paraformaldehyde. The brains were removed and $20-\mu \mathrm{m}$ frozen sections were prepared. ChAT immunoreactivity was identified using a rat antiserum to pig ChAT prepared essentially as described previously (Eckenstein et al., 1981; Eckenstein and Thoenen, 1982). Free-floating $20-\mu \mathrm{m}$ sections were incubated overnight in 1:500 diluted antiserum at $4^{\circ} \mathrm{C}$, washed, and incubated for $1 \mathrm{hr}$ in 1:50 diluted fluorescein isothiocyanate-labeled goat anti-rat antibodies as previously described (Eckenstein and Thoenen, 1982). After washing, the sections were mounted on gelatinized slides and fixed in place by brief application of a filter paper saturated with $4 \%$ paraformaldehyde. The slides were coverslipped with aqueous medium and selected areas were photographed with a Leitz fluorescent microscope. The coverslips were then carefully removed, and the sections were processed for AChE histochemistry using the thiocholine procedure (Lewis and Shute, 1969). They were subsequently dehydrated and coverslipped, and the same selected areas were rephotographed with Zeiss brightfield optics. For all regions of the forebrain described, 100 to 500 neurons were analyzed by comparison of photomicrographs. To establish that the observed differential staining was not due to a difference in focus depth between the two types of photographs, in one experiment the immunofluorescence was photographed at different foci, without yielding altered results.

The specificity of the ChAT staining observed was tested as previously described (Eckenstein et al., 1981; Eckenstein and Thoenen, 1982; Sofroniew et al., 1982) and by preabsorption of the antiserum with pig ChAT coupled to Sepharose 4B (Pharmacia) which prevented staining of neurons. Specificity of the AChE staining observed was tested by addition of $10^{-5} \mathrm{M}$ eserine to the incubation medium which prevented $\mathrm{AChE}$ staining of neurons.

\section{Results}

Comparison of neighboring sections stained alternately for ChAT or AChE indicated that, in those areas previously described as containing ChAT-immunoreactive neurons (Sofroniew et al., 1982), AChE-positive neurons were also present, whereas a number of areas appeared to contain only AChE-positive neurons. In the regions of the brain examined, there were no obvious areas containing only ChAT-positive neurons and no AChE-positive neurons. Pretreatment of the animals with colchicine did not appear to alter the number of neurons stained positively for ChAT or the overall distribution of areas in which ChAT-positive neurons were found. Based on examination of neighboring sections, forebrain areas which contained both ChAT-positive and AChE-positive neurons included the tuberculum olfactorium, the nucleus accumbens, the islands of Calleja, the nucleus of the diagonal band, the septum, the basal forebrain area around the medial forebrain bundle, the neostriatum, and the nucleus basalis of Muynert. Based on examination of neighboring sections, areas which contained AChE-positive neurons but obviously did not contain any ChAT-positive neurons in normal or colchi- 
cine-treated rats included the hypothalamic arcuate and dorsomedial nuclei, lateral posterior hypothalamus, zona incerta, and substantia nigra.

In order to test more exactly at the single cell level whether the individual neurons in a given area contained both ChAT and AChE, a dual localization procedure was developed using immunofluorescence staining for ChAT, photography of neurons in a selected area, reprocessing the same sections for $\mathrm{AChE}$ histochemistry, and rephotography of the same neurons. In this manner several hundred neurons were compared in each of five areas selected as representative on the basis of the neighboring section comparisons conducted previously. These areas were the nucleus of the diagonal band of Broca (vertical and horizontal limbs), the septum, the caudate putamen, the nucleus basalis of Meynert, and the zona incerta. The rats used for this direct comparison were pretreated with DFP, an irreversible AChE inhibitor, and allowed to survive for $8 \mathrm{hr}$ prior to fixation. This treatment allowed the clear and distinct visualization of cell bodies containing newly synthesized $\mathrm{AChE}$ without interference of the often very intense AChE staining of the surrounding neuropil which often obscures cell bodies (Butcher, 1978; Fibiger, 1982). Pretreatment with DFP had no discernible effect on the number, distribution, or staining intensity of ChAT-positive neurons. The findings obtained in the five areas examined in detail are as follows.

Caudate putamen. Here, the histochemical stain for AChE showed complete overlap with the immunofluorescence label for ChAT (Fig. 1), indicating that all AChE-positive neurons in this area also contain ChAT and vice versa. This appeared to include both the large (20 to $40 \mu \mathrm{m}$ ) and small (10 to $20 \mu \mathrm{m}$ ) AChE-positive neurons which have been reported to be distinguishable in this nucleus (Henderson, 1981; Woolf and Butcher, 1981), although we could not distinguish between two populations of neurons. In the $20-\mu \mathrm{m}$ sections used for the double-labeling studies we felt we could not be certain that the smaller profiles did not simply represent angled sections of larger neurons, since in 50- and $100-\mu \mathrm{m}$-thick sections of striatum we have found ChAT-positive neurons to have fairly heterogenous shapes with neurons having small diameters as short as $12 \mu \mathrm{m}$ or large diameters as long as $40 \mu \mathrm{m}$ and a continuous range of sizes in between. Most neurons appear to have at least one diameter of about $20 \mu \mathrm{m}$ or more and they appear to constitute a single population of neurons which we cannot, at present, obviously divide into distinct subsets solely on the basis of size. This observation is supported by the recent report that a statistical analysis of the size of striatal AChE-positive neurons indicates that there is only a single population (Fibiger, 1982, quoting J. Lehmann and H. C. Fibiger, manuscript in preparation).

Nucleus basalis magnocellularis of Meynert. This nucleus is defined according to previous descriptions (Lehmann et al., 1980; Bigl et al., 1982; Fibiger, 1982) and in the rat, to a large extent, comprises the large neurons medial to the globus pallidus intermingled in clusters of white matter. All AChE-positive neurons of the nucleus basalis were also positive for ChAT and vice versa (Fig. 2 ). The large, intensely AChE-positive neurons of the basal nucleus were easily distinguished from the many
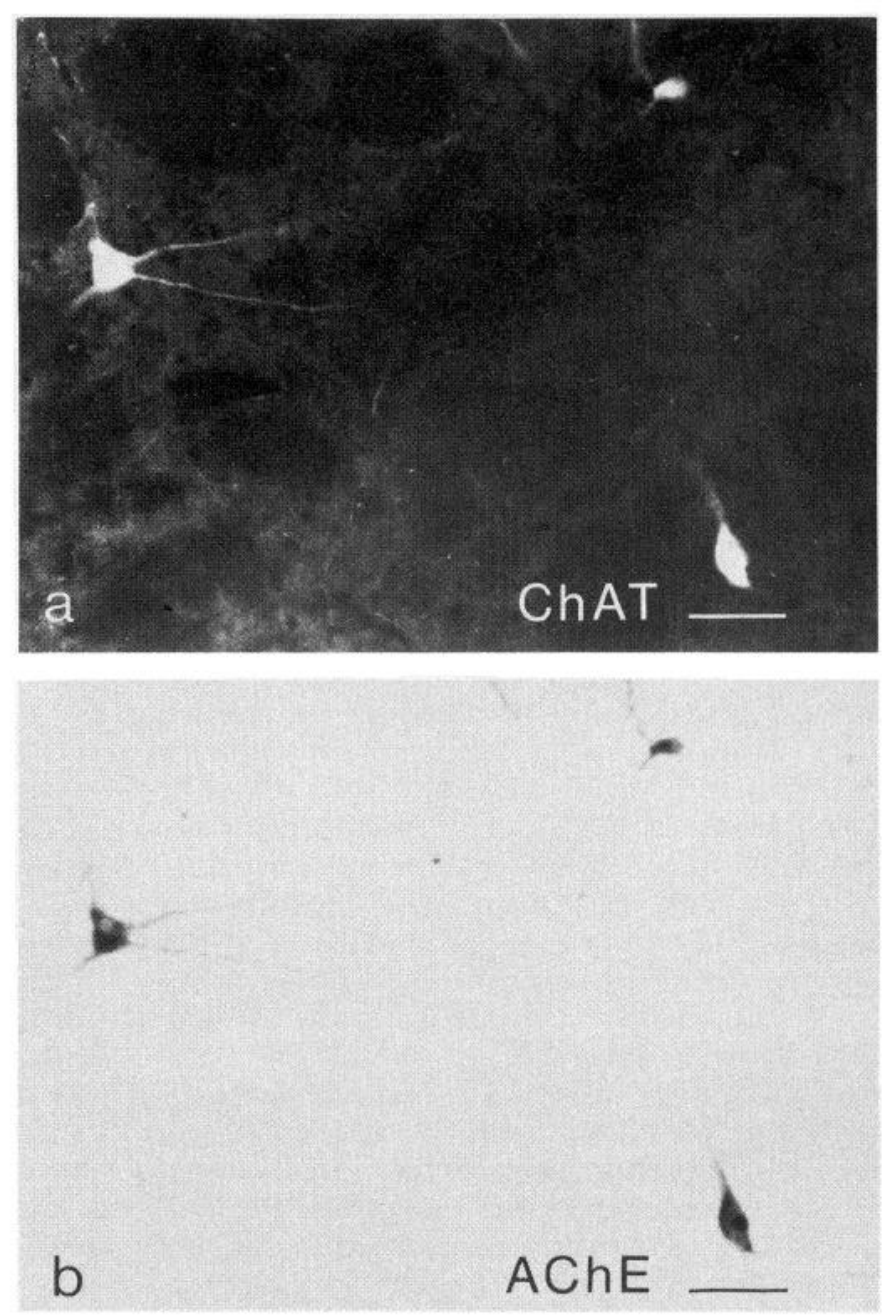

Figure 1. Caudate putamen of a DFP-treated rat. The same $20-\mu \mathrm{m}$ frontal frozen section has been stained by the immunofluorescence procedure for ChAT and photographed $(a)$, then processed for $\mathrm{AChE}$ activity by the thiocholine procedure, and the same area of the caudate putamen was then rephotographed (b). Note that all neurons positive for ChAT are also positive for AChE and vice versa. Scale bar $=50 \mu \mathrm{m}$.

smaller, less intensely AChE-positive neurons in the globus pallidus and vicinity, none of which were ChAT positive.

Medial septum and nucleus of the diagonal band of Broca (including vertical and horizontal limbs). These areas are considered together because similar observations were made in both. Here, all ChAT-positive neurons contained $\mathrm{AChE}$, but these neurons containing both ChAT and AChE were intermingled with a smaller number of neurons positive only for AChE (Figs. 3 and 4). It was not possible to distinguish between these two types of AChE-positive neurons on the basis of their morphology or intensity of AChE staining. Throughout these two areas, approximately $12 \%$ of the intensely AChE-positive neurons did not contain ChAT, judged by comparing photomicrographs of several hundred neurons in doublestained sections (as in Figs. 3 and 4) through several representative levels. No neurons containing only ChAT were observed, although some (less than 5\%) ChAT neurons were only weakly positive for AChE (Fig. 4). 

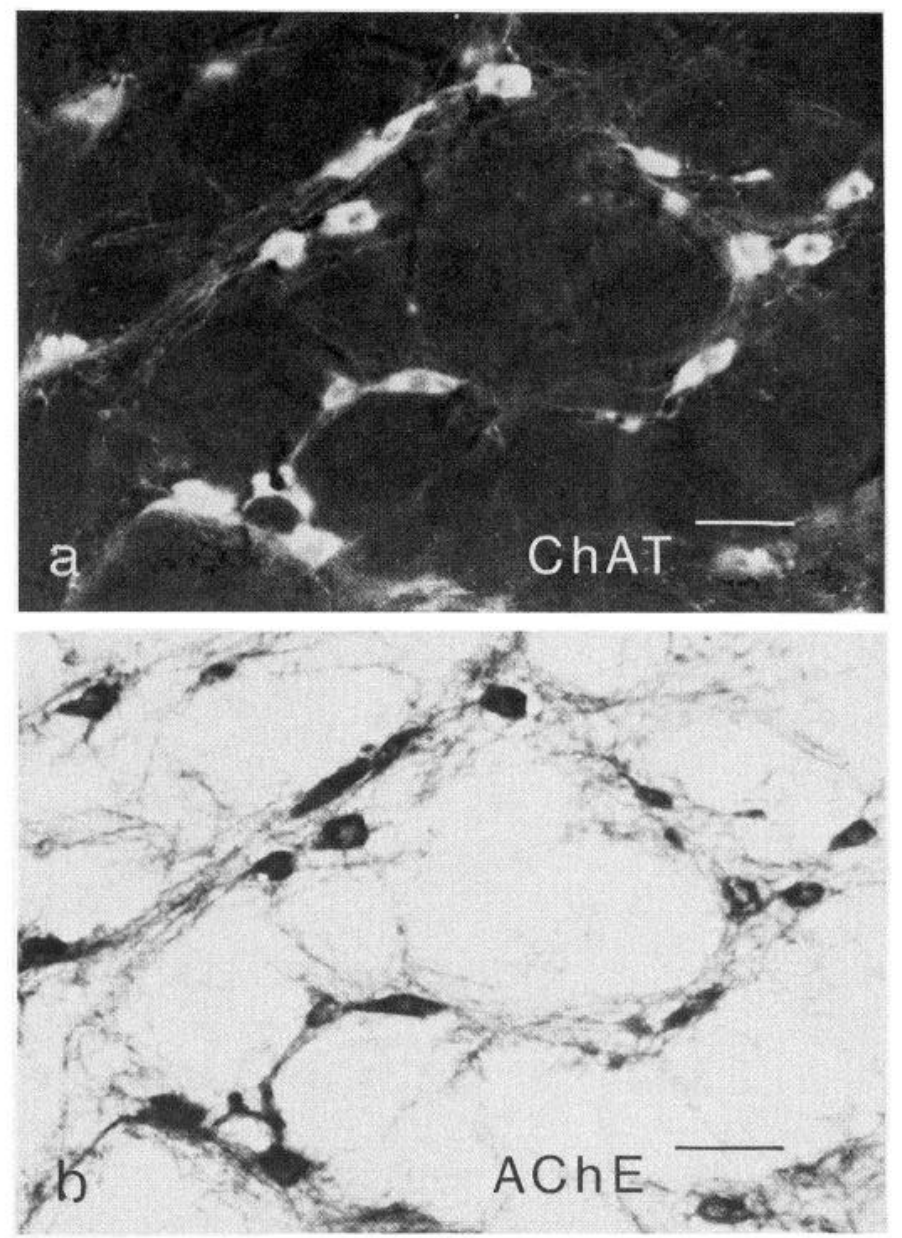

Figure 2. Nucleus basalis magnocellularis of Meynert of a DFP-treated rat. The same $20-\mu \mathrm{m}$ frontal frozen section has been stained by the immunofluorescence procedure for ChAT and photographed $(a)$, then processed for AChE activity by the thiocholine procedure, and the same area of the nucleus basalis magnocellularis of Meynert was then rephotographed $(b)$. Note that all neurons positive for ChAT are also positive for AChE and vice versa. Scale bar $=50 \mu \mathrm{m}$.

Zona incerta. No ChAT-immunoreactive neurons were ever found in this region although there were always numerous AChE-positive neurons present (Fig. 5).

\section{Discussion}

In this study we have demonstrated that certain neurons contain both ChAT, the cholinergic transmittersynthesizing enzyme, and $\mathrm{AChE}$, the transmitter-degrading enzyme. In addition, neurons were found containing the transmitter-degrading enzyme $\mathrm{AChE}$, in the apparent absence of the transmitter-synthesizing enzyme ChAT, whereas the reverse case of ChAT without $\mathrm{AChE}$ was not observed. These findings strongly suggest that central cholinergic neurons can be characterized by their expression of both $\mathrm{ChAT}$ and $\mathrm{AChE}$. In addition, other, apparently noncholinergic neurons exist which express AChE but not ChAT. The validity of this conclusion is supported by the findings presented here that certain neurons containing AChE do not contain immunoreactive ChAT in normal or in colchicine-treated rats. Col-
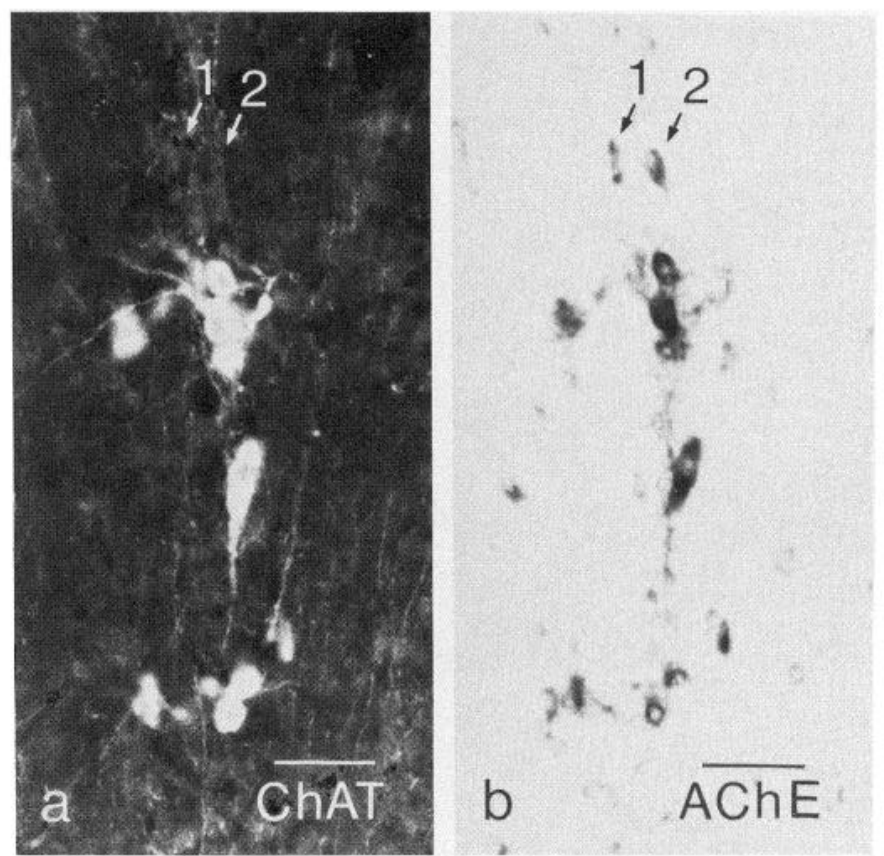

Figure 3. Medial septum of a DFP-treated rat. The same 20$\mu \mathrm{m}$ frontal frozen section has been stained by the immunofluorescence procedure for ChAT and photographed $(a)$, then processed for $\mathrm{AChE}$ activity by the thiocholine procedure, and the same area of the medial septum was then rephotographed (b). Note that, although all neurons positive for ChAT are also positive for $\mathrm{AChE}$, two neurons ( 1 and 2 ) intensely positive for AChE are not positive for ChAT. Scale bar $=50 \mu \mathrm{m}$.

chicine is known to block axonal transport (Dahlström, 1968; Kreutzberg, 1969) and is regularly used in immunohistochemical studies to visualize neuronal perikarya not visible in untreated animals. In addition, the ChAT antisera used precipitate all of the ChAT activity in rat brain, making it unlikely that an additional molecular form of the enzyme exists which has not been recognized. Furthermore, our double-labeling procedure for visualization of both ChAT and AChE in the same neurons in the same sections combines the use of fluorescent and brightfield techniques. This allows each to be carried out at maximum intensity without the interference during evaluation which would be expected using two procedures which both require brightfield evaluation. Thus, it seems unlikely that we have failed to visualize all ChAT or all $\mathrm{AChE}$ neurons in a compromise for the simultaneous localization procedure. These findings thus demonstrate at the cellular level that the histochemical stain for $\mathrm{AChE}$ cannot be considered an unambiguous marker for cholinergic neurons. Indeed, examples already exist of the identification of $\mathrm{AChE}$ in monoaminergic neurons in the substantia nigra and locus ceruleus, and available biochemical evidence indicates that these neurons are not cholinergic (see Butcher, 1978; Fibiger, 1982).

It has been suggested that neurons intensely stained for AChE following DFP treatment are more likely to be cholinergic, and this criterion is used by some investigators (see Butcher, 1978; Fibiger, 1982). However, we did not find a constant correlation between intensity of AChE staining following DFP treatment and ChAT con- 

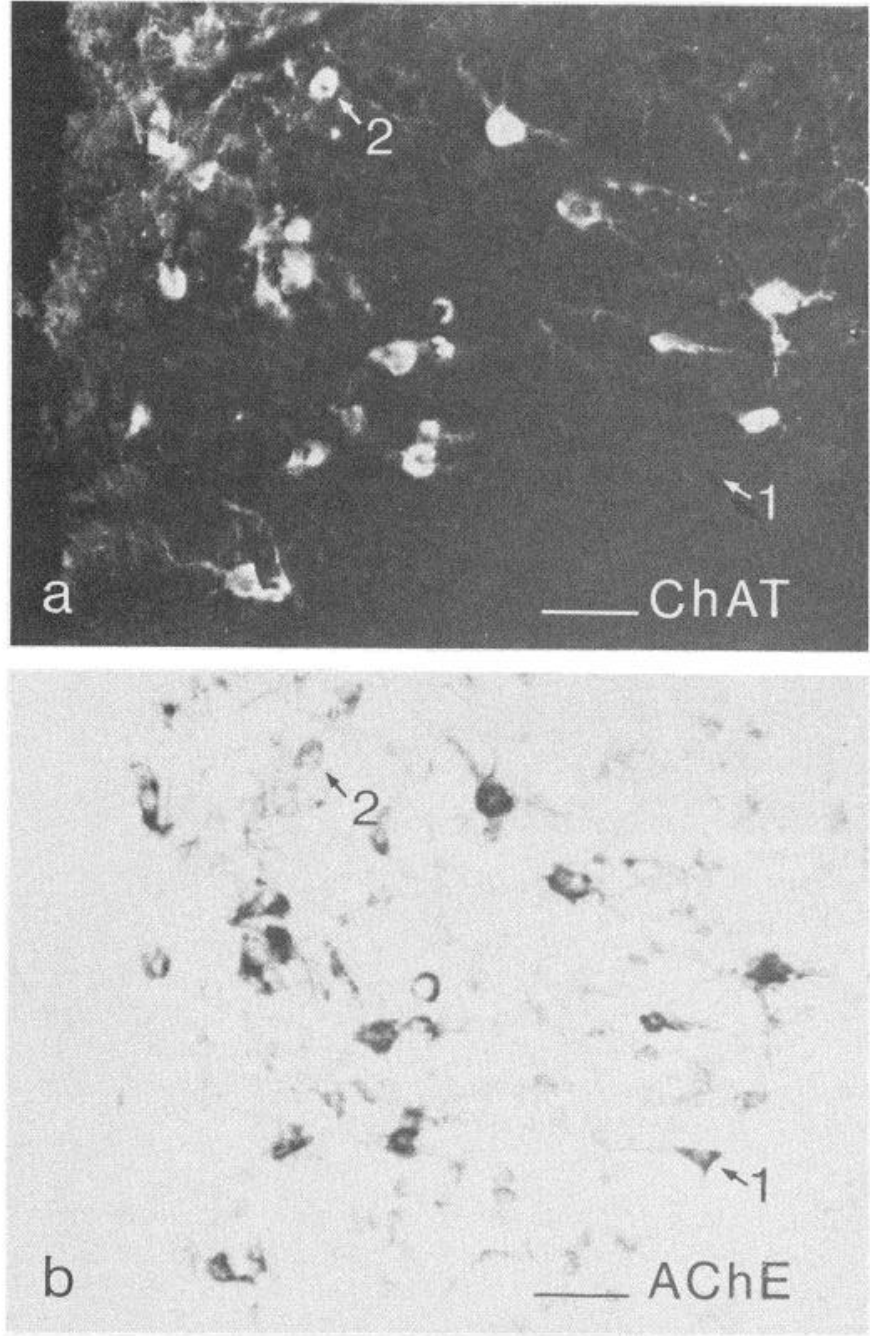

Figure 4. Nucleus of the diagonal band of Broca of a DFPtreated rat. The same $20-\mu \mathrm{m}$ frontal frozen section has been stained by the immunofluorescence procedure for ChAT and photographed (a), then processed for AChE activity by the thiocholine procedure, and the same area of the nucleus of the diagonal band of Broca was then rephotographed $(b)$. Note that, although all neurons positive for ChAT are also positive for $\mathrm{AChE}$, one neuron (1) intensely positive for AChE is not positive for ChAT. Note also that one neuron (2) clearly positive for ChAT is only very weakly positive for AChE. Scale bar $=50 \mu \mathrm{m}$.

tent of the neurons. Many so-called intensely stained AChE neurons did not contain ChAT, and in some cases ChAT-positive neurons were only weakly stained for AChE (Figs. 3 and 4). It is unlikely that these observations are due to technical artifacts since, as described above, our double-labeling procedure allows the reactions for both ChAT and AChE visualization to be carried out at maximum intensity. Nevertheless, the AChE procedure, which is simple, reproducible, and has found widespread application (Butcher, 1978; Fibiger, 1982), may be of use in identifying cholinergic neurons in certain areas. In two of the areas examined in this study, the caudate putamen and nucleus basalis magnocellularis of Meynert, there was $100 \%$ congruence between ChAT-
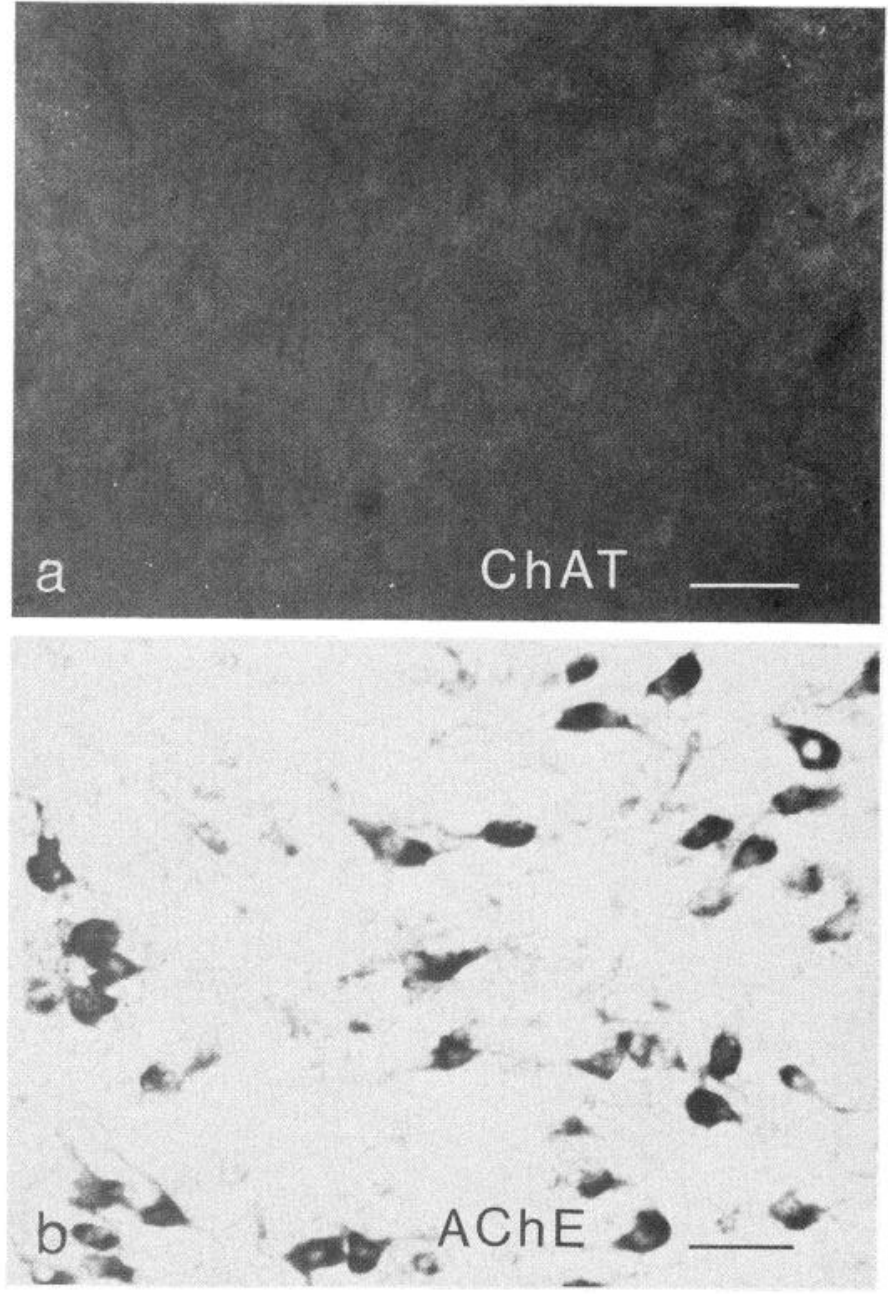

Figure 5. Zona incerta of a DFP-treated rat. The same 20$\mu \mathrm{m}$ frontal frozen section has been stained by the immunofluorescence procedure for ChAT and photographed $(a)$, then processed for $\mathrm{AChE}$ activity by the thiocholine procedure, and the same area of the zona incerta was then rephotographed $(b)$. Note that, although this area contains many neurons intensely positive for AChE $(b)$, none of these neurons are positive for ChAT $(a)$. Scale bar $=40 \mu \mathrm{m}$.

positive and AChE-positive neurons. However, in two other regions, the septum and diagonal band, neurons positive for both ChAT and AChE were intermingled with less frequently occurring neurons positive only for $\mathrm{AChE}$. This indicates that in these regions the presence of $\mathrm{AChE}$ (even of so-called intensely positive $\mathrm{AChE}$ ) is not an absolute marker for cholinergic neurons, even though many ChAT-positive neurons have been identified there. This observation demonstrates the value of the method used, as only a direct anatomical analysis at the cellular level is capable of demonstrating such intermingled populations. It also indicates the necessity of examining all newly studied regions in a similar manner without assuming that overlap of ChAT and AChE in a given area, even in neighboring sections, can be interpreted as cell-to-cell correspondence.

Studies combining the biochemical deteminations of ChAT and AChE with lesions have suggested that cho- 
linergic neurons express both enzymes (Lehmann et al., 1980). This is in agreement with our findings that, in the areas examined, all ChAT-positive neurons also contained AChE. There are a number of possible reasons why a neuron synthesizing a transmitter might also produce an enzyme for the degradation of that transmitter. The reasons underlying the large number of presumptive noncholinergic neurons which contain AChE but not ChAT are less clear. These neurons may be cholinoreceptive and contain the transmitter-degrading enzyme for this reason. However, many of these neurons are present in areas which do not appear to contain many cholinergic terminals. Another alternative is that AChE may be involved in activities not related to acetylcholine, such as degradation of peptides (Chubb et al., 1980) or other activities (Greenfield et al., 1981).

\section{References}

Bigl, V., N. J. Woolf, and L. L. Butcher (1982) Cholinergic projections from the basal forebrain to frontal, parietal, temporal, occipital, and cingulate cortices: A combined fluorescent tracer and acetylcholinesterase analysis. Brain Res. Bull. 8: 727-749.

Butcher, L. L. (1978) Recent advances in histochemical techniques for the study of central cholinergic mechanisms. In Cholinergic Mechanisms and Psychopharmacology, D. J. Jenden, ed., pp. 93-124, Plenum Press, New York.

Chubb, I. W., A. J. Hodgson, and G. H. White (1980) Acetylcholinesterase hydrolyzes substance P. Neuroscience 5: 20652072.

Dahlström, A. (1968) Effect of colchicine on transport of amine storage granules in sympathetic nerves of rat. Eur. J. Pharmacol. 5: 111-112.

Eckenstein, F., and H. Thoenen (1982) Production of specific antisera and monoclonal antibodies to choline acetyltrans ferase: Characterization and use for identification of cholinergic neurons. EMBO J. 1: 363-368.

Eckenstein, F., Y. A. Barde, and H. Thoenen (1981) Production of specific antibodies to choline acetyltransferase purified from pig brain. Neuroscience 6: 993-1000.

Fibiger, H. C. (1982) The organization and some projections of cholinergic neurons of the mammalian forebrain. Brain Res.
Rev. 4: 327-388.

Fonnum, F. (1975) Review of recent progress in the synthesis, storage, and release of acetylcholine. In Cholinergic Mechanisms, P. G. Waser, ed., pp. 145-160, Raven Press, New York.

Greenfield, S. A., J. F. Stein, A. J. Hodgson, and I. W. Chubb (1981) Depression of nigral pars compacta cell discharge by exogenous acetylcholinesterase. Neuroscience 6: 2287-2295.

Henderson, Z. (1981) Ultrastructure and acetylcholinesterase content of neurones forming connections between the striatum and substantia nigra of rat. J. Comp. Neurol. 197: 185196.

Jacobowitz, D. M., and M. Palkovits (1974) Topographic atlas of catecholamine and acetylcholinesterase-containing neurons in the brain. I. Forebrain (telencephalon, diencephalon). J. Comp. Neurol. 157: 13-28.

Koelle, G. B., and J. S. Friedenwald (1949) A histochemical method for localizing cholinesterase activity. Proc. Soc. Exp. Biol. Med. 70: 617-622.

Kreutzberg, G. (1969) Neuronal dynamics and flow. IV. Blockage of intra-axonal enzyme transport by colchicine. Proc. Natl. Acad. Sci. U. S. A. 62: 722-728.

Lehmann, J., J. I. Nagy, S. Atmadja, and H. C. Fibiger (1980) The nucleus basalis magnocellularis: The origin of a cholinergic projection to the neocortex of the rat. Neuroscience 5 : 1161-1174.

Lewis, P. R., and C. C. D. Shute (1969) An electron microscopic study of cholinesterase distribution in the rat adrenal medulla. J. Microsc. 89: 181-193.

Rossier, J. (1977) Choline acetyltransferase: A review with special reference to its cellular and subcellular localization. Int. Rev. Neurobiol. 20: 284-337.

Rossier, J. (1981) Serum monospecificity: A prerequisite for reliable immunohistochemical localization of neuronal markers including choline acetyltransferase. Neuroscience 6: 989 991.

Sofroniew, M. V., F. Eckenstein, H. Thoenen, and A. C. Cuello (1982) Topography of choline acetyltransferase-containing neurons in the forebrain of the rat. Neurosci. Lett. 33: 7712.

Woolf, N. J., and L. L. Butcher (1981) Cholinergic neurons in the caudate-putamen complex proper are intrinsically organized: A combined Evans blue and acetylcholinesterase analysis. Brain Res. Bull. 7: 487-507. 